\title{
An energy efficient clustering time synch algorithm for WSN in bridge health diagnosis system
}

\author{
Haitao Xiao ${ }^{\text {a) }}$, Harutoshi Ogai, and Noriyoshi Yamauchi \\ Graduate School of Information, Production and systems, Waseda University \\ 2-7 Hibijino, Wakamatsu-ku, Kitakyushu, Fukuoka 808-0135, Japan \\ a)xiaohaitao@fuji.waseda.jp
}

\begin{abstract}
This paper describes a novel time synchronization algorithm for the WSN of bridge health diagnosis system. In previous work, we develop a time synchronization algorithm, named DMLTS [1], for this WSN. To reduce energy consumption, this time synchronization improves the time-synch phase of DMLTS and utilizes an improved energy feedback scheme to lengthen the lifetime of WSN and reduce synchronization error. Performance analyses, simulations and realization in node hardware of WSN are also presented. They proved that: this algorithm has a better synchronization error performance, can decrease overhead, consume less energy, and lengthen the lifetime of WSN.
\end{abstract}

Keywords: time synchronization, energy feedback, bridge diagnosis Classification: Transmission Systems and Transmission Equipment for Communications

\section{References}

[1] H. T. Xiao, H. Ogai, and Z. H. Ding, "A Distributed Multi-hop Low Cost Time Synchronization Algorithm in Wireless Sensor Network developed for Bridge Diagnosis System," IEEJ Transactions on Electronics, Information and Systems, vol. 132, no. 5, pp. 656-665, May 2012.

[2] H. T. Xiao and H. Ogai, "A Self-Health Monitoring System for a Wireless Sensor Network Used in Bridge Diagnosis," SICE Journal of Control, Measurement, and System Integration, vol. 5, no. 1, pp. 48-54, Jan. 2012.

[3] W. R. Heinzelman and H. Balakrishnan, "An application-specific protocol architecture for wireless microsensor networks," IEEE Trans. Wireless Commun., vol. 1, no. 4, pp. 660-670, Oct. 2002.

[4] S. Ganeriwal and R. Kumar, "Timing-sync protocol for sensor networks," Proc. 1th International Conference on Embeded Networked Sensor Systems, Los Angele CA, USA, pp. 138-149, 2003.

\section{Introduction}

Due to the mostly mountainous and rugged landform, and frequent earthquakes in Japan, bridge health diagnosis is a problem of greater complexity. 
In the bridge health diagnosis system (BHD), we develop a WSN to sample and gather the vibration data of bridge. Since time synchronization provides a fundamental service for building many applications, it is critical for the WSN. For example inBHD, sensor nodes need a common timestamp to sample the vibration, and nodes must sleep and wake up in the same time to achieve comparable detected data.

In previous work, we develop a time synchronization algorithm, named DMLTS [1], for this WSN. DMLTS combines the advantages of RBS and TPSN to reduce the number of exchanging packets during synchronization. In each cluster, it uses an energy feedback scheme to select a messenger node to implement pairwise synchronization with head node. Then, the head node broadcasts the result of this pairwise synchronization to its child nodes to adjust their clock. [1] gives the performance analyses, simulations and realization of DMLTS, comparing with RBS, TPSN and FTSP. The problem of DMLTS is that: Frist, the energy of head node is consumed too large. It will shorten the life time of WSN. Second, if the communication condition between head node and the messenger node is not very good, the synchronization packet loss will occur between them during the pairwise synchronization. It makes this round of synchronization fail and lads to consume more energy. To solve these problems, Energy-efficient Clustering Time Synchronization algorithm (ECTS) is proposed. ECTS is based on DMLTS and an improved energy feedback scheme.

\section{Energy-efficient clustering time synchronization algorithm}

The ECTS algorithm contains 2 phases: establishing of spanning tree phase and time synchronization phase. In single-hop WSN it only contains the time synchronization phase. In multi-hop WSN, the establishing of spanning tree phase of ECTS is the same as the DMLTS.

\subsection{Time synchronization in single-hop WSN}

Time synchronization process of DMLTS and ECTS are shown in Fig. 1. In DMLTS, at first the root node broadcasts a synchronization packet $M 1$ to its child node 1, 2 and $k$ and $M 1$ contains Node 1's identifier for example, and recode the send time $[t 1]_{R N}^{1}$. That means Node 1 is chose as "messenger node" (2.2 will describe how to choose the messenger node). In actually all child nodes of root node will receive $M 1$. After Node 1 gets this packet, it knows the identifier in the packet is the same as its own. Node 1 records the receiving time $[t 2]_{N 1}^{1}$. During a very short time, before Node 1 sends back a packet $M 2$ to the root node, it records that instantaneous time $[t 3]_{N 1}^{1}$ and puts the $[t 2]_{N 1}^{1}$ and $[t 3]_{N 1}^{1}$ in $M 2$. When the root node receives $M 2$ it also records the receiving time $[t 4]_{R N}^{1}$. So root node can calculate time offset with Node 1 as shown in Eq. (1), where $O_{R, N 1}$ indicates the time offset. At last root node will broadcast a packet $M 3$ contained $O_{R, N 1}$ and the Node 1's time $[t 2]_{N 1}^{1}$. Node 1 adjusts its clock based on $O_{R, N 1}$. The other nodes calculate their time offset and adjust their clock based on Eq. (2) and Eq. (3), i.e. 


\section{DMLTS algorithm}
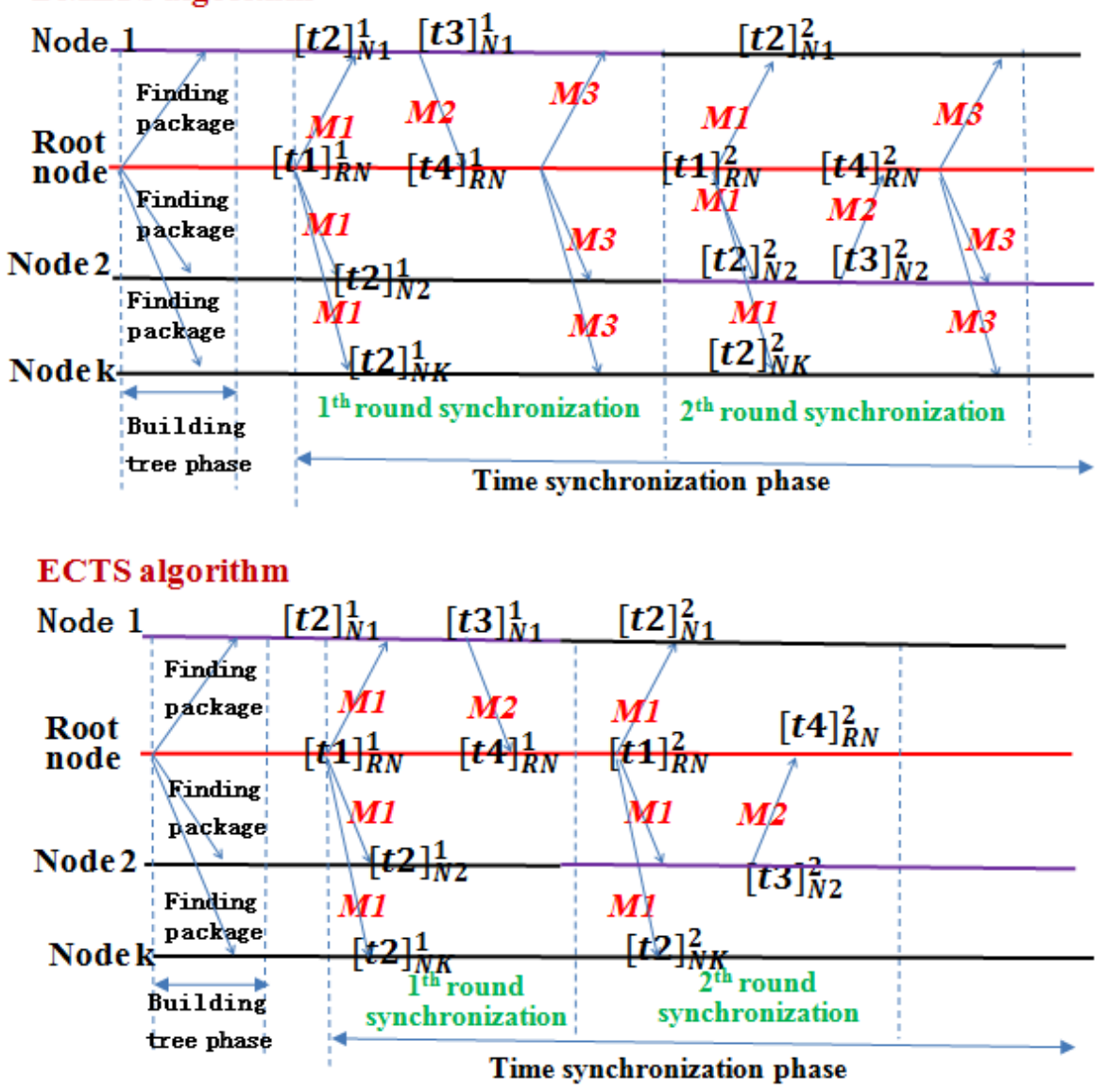

Fig. 1. Process of DMLTS and ECTS

to Node $K$. After adjustment, WSN go to $2^{\text {th }}$ round time synchronization, which is the same as $1^{\text {th }}$ round.

$$
\begin{aligned}
& O_{R, N 1}=\frac{\left([t 2]_{N 1}^{1}-[t 1]_{R N}^{1}\right)-\left([t 4]_{R N}^{1}-[t 3]_{N 1}^{1}\right)}{2} . \\
& {[t 2]_{R N}^{1}+O_{R, N 1}=[t 2]_{N K}^{1}+O_{N 1, N K} .} \\
& O_{R, N K}=O_{R, N 1}-\left([t 2]_{N 1}^{1}-[t 2]_{N K}^{1}\right) .
\end{aligned}
$$

ECTS modifies DMLTS in two aspects. First, in ECTS, the root node does not broadcast $M 3$ again. The information of $M 3$ will be piggybacked in $M 1$ of the next round as shown in Fig. 1. There are only two packets in one synchronization round. So when a node receives $M 1$, it not only calculates the offset time and adjusts clock for last round but also records time information for this round. Second, we improve the energy feedback scheme (it is used to choose the messenger node) to improve synchronization error and avoid the packet loss between root node and messenger node, which is caused by weakly quality of link. The improved energy feedback scheme will be described in section 2.2 .

\subsection{Messenger node choosing scheme}

In each synchronization round, the message node will consume more energy and always has a more accurate time clock than other child nodes in DMLTS, 
since it direct implement pair synchronization with root node. To length node's life and improved the synchronization error, we proposed an energy feedback scheme shown in Eq. (4), where $E_{\text {rest }}$ means the rest energy of the node and $E_{i n i}$ is the original energy of the node. $\varepsilon$ is a historical record factor: if one node is chosen as the message node this time, its $\varepsilon$ value will be set to 0 , otherwise this value is set to 1 . The node had the biggest $\pi_{\text {energy }}$ will be chosen as messenger node.

$$
\pi_{\text {energy }}=\frac{\varepsilon E_{\text {rest }}}{E_{\text {ini }}} .
$$

In ECTS, to solve the second problem of DMLTS described in section 1, we improved the energy feedback $\pi_{\text {energy }}$. The improved energy feedback equation is shown in Eq. (5), where, $\alpha$ is a communication condition factor. When the RSSI of node is greater than a threshold (it is $-90 \mathrm{dbm}$ in the WSN of BHD), which means the wireless communication condition is good, $\alpha$ will be set to 1 , otherwise it will be set to 0 . The communication condition factor $\alpha$ ensure the quality of link between root node and messenger node is good. Thus it reduces the probability of synchronization packet loss. We must notice that if all the RSSI of nodes are less than the threshold, all nodes' $\alpha$ are set to 1 . In multi-hop network, to reduce the synchronization error caused by multi-hop, we need to select the node contained more child nodes as messenger node. Thus a factor $\left(N_{\text {self }} / N_{\text {all }}\right)$ is used to improve the energy feedback equation. $N_{\text {self }}$ is the number of node's child nodes, and $N_{\text {all }}$ is the number of all the nodes' child nodes of this cluster. Thus the biggest $\pi_{\text {energy }}$ will be chosen as messenger node.

$$
\pi_{\text {energy }}=\varepsilon \alpha\left(\frac{E_{\text {rest }}}{E_{\text {ini }}}+\frac{N_{\text {self }}}{N_{\text {all }}}\right) .
$$

\subsection{Time synchronization in multi-hop WSN}

In multi-hop WSN the spanning tree and clusters are established in establishing of spanning tree phase. Since the spanning tree and cluster are established and divided by seal-monitoring system (DLDMS) [2] in BDH, ECTS does not need establishing of spanning tree phase here. The spanning tree of this WSN is shown in Fig. 2. After establish spanning tree and clusters, the

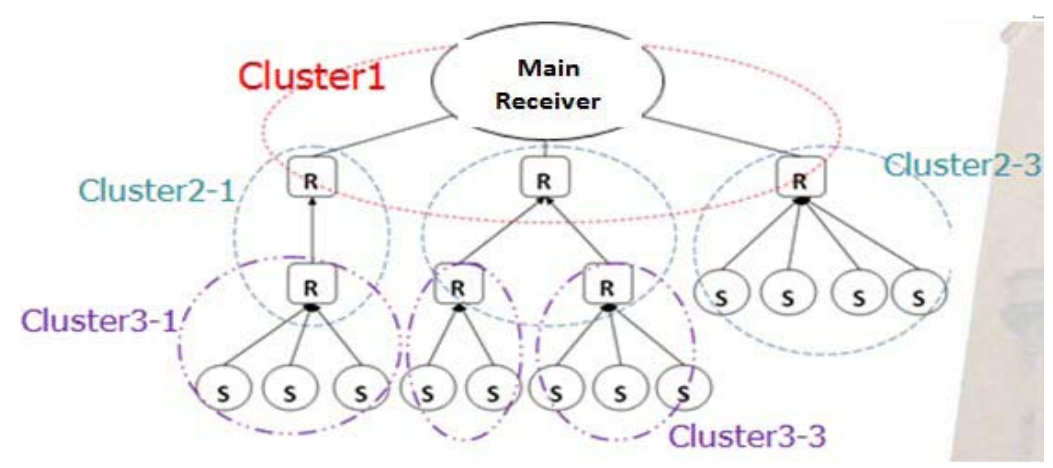


ECTS is applied in each cluster as the same as using in single-hop WSN. In each cluster, the head node is regarded as root node. The low level clusters implement time synchronization before high level clusters.

\section{Performance evaluation and simulation}

[1] gives a performance evaluation of DMLTS by comparing with RBS, TPSN and FTSP. The result shows the energy consumption of DMLTS is far small

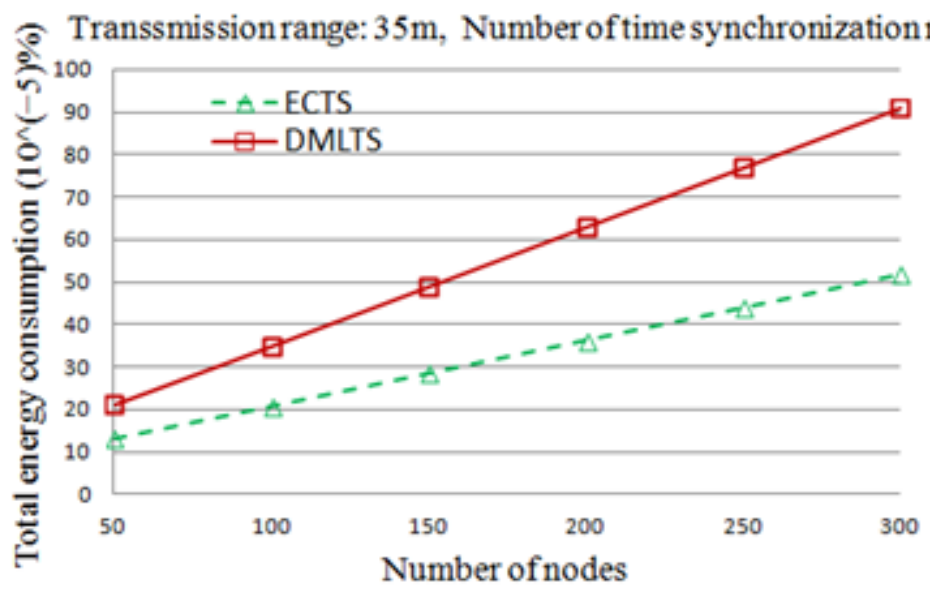

(a) Total energy consumption VS Number of nodes

Namber of nodes: 100, Number of time synchronization round : 10

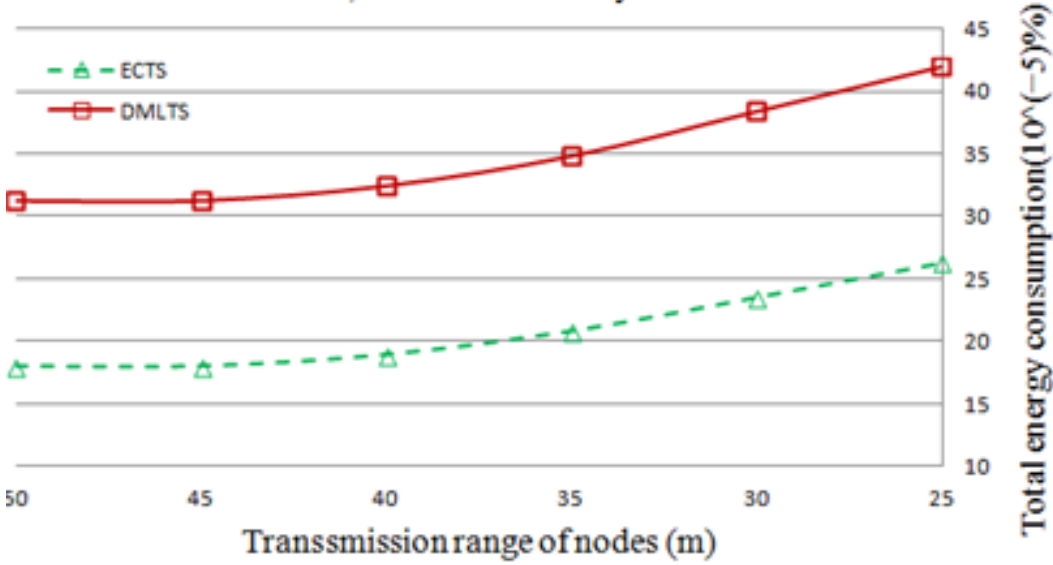

(b) Total energy consumption VS Transmission range of nodes

Synchronization error in single-hop WSN

\begin{tabular}{|c|c|c|}
\hline Algorithm & $\mu(\mu \mathrm{s})$ & Required timing massagers \\
\hline DMLTS & 21.62 & 3 \\
\hline ECTS & 20.39 & 2 \\
\hline
\end{tabular}

Synchronization error in multi-hop WSN

\begin{tabular}{|c|c|c|}
\hline Hop number & DMLTS (us) & ECTS (us) \\
\hline 1 & 21.93 & 20.67 \\
\hline 2 & 35.26 & 29.56 \\
\hline 3 & 47.38 & 40.75 \\
\hline
\end{tabular}

(c) Synchronization error 
than others, and the synchronization error is within dozens of microseconds. Thus we evaluate the performance of ECTS by comparing with DMLTS here. In each cluster, compared with ECTS, DMLTS requires cluster head broadcasts extra one packet for each round, and child nodes need to receive extra one packet. Fig. 3 (a) shows the simulation result of the energy consumption of ECTS and DMLTS. In this simulation, the nodes are randomly deployed on an area of $100 \mathrm{~m} * 100 \mathrm{~m}$, and the root node is assumed to be located at the center of the simulation area. The number of synchronization rounds is 10 and the transmission range of nodes is $35 \mathrm{~m}$. Since the energy consumption of receiving a message is almost $40 \%$ of sending a message in the WSN of $\mathrm{BHD}$, we set the energy consumption of receiving is $40 \%$ of sending here. It can be seen that the energy consumption of ECTS is less than DMLTS obviously. Fig. 3 (b) evaluates the performance of ECTS with respect to the transmission range of nodes assuming the same simulation setup as in the previous figure. The number of overall nodes is fixed to 100 in this simulation. It can be seen that as the transmission range reduce (the number of cluster increase), ECTS save more energy than DMLTS. From Fig.3(c), the time synchronization is the same almost in single-hop WSN. But in multi-hop WSN, ECTS is better than DMLTS obviously in high level clusters.

\section{Conclusion}

In this paper we propose an improved time synchronization algorithm based on DMLTS and an energy feedback scheme. This algorithm reduces the energy consumption of head node of clusters by using a broadcast synchronization concept. In the same time we design an energy feedback scheme to reduce the multi-hop synchronization error and lengthen the lifetime of the WSN. The simulations prove our proposed approach has a better performance in synchronization error and energy consumption, comparing with DMLTS. This timing synchronization algorithm had been used in the wireless sensor network of bridge diagnosis system.

\section{Acknowledgments}

This study receives support from Japan Regional Innovation Strategy Program and financial support from Health Monitoring Business Limited Liability Partnership. 